\title{
Time change approach to generalized excursion measures, and its application to limit theorems
}

Patrick J. Fitzsimmons and Kouji Yano*

Dedicated to Professor M. Fukushima on the occasion of his seventieth birthday.

\begin{abstract}
It is proved that generalized excursion measures can be constructed via time change of Itô's Brownian excursion measure. A tightness-like condition on strings is introduced to prove a convergence theorem of generalized excursion measures. The convergence theorem is applied to obtain a conditional limit theorem, a kind of invariance principle where the limit is the Bessel meander.
\end{abstract}

\section{Introduction}

Stone [19] has proved various limit theorems for Markov processes via time change of Brownian motion. The condition assumed is the pointwise convergence of strings. Recently Kotani [13] and Kasahara-Watanabe [12] have posed a tightness-like condition to study the scaling limit of the fluctuation of various degenerate limits. The second author 23] has studied this problem from the viewpoint of generalized excursion measures through a spectral theoretic approach.

In the present paper we construct generalized excursion measures via time change of Itô's Brownian excursion measure. We introduce a weaker tightness-like condition than Kotani and Kasahara-Watanabe's and prove a convergence theorem for generalized excursion measures. We apply the convergence theorem to generalize the conditional limit theorem obtained by Li-Shiga-Tomisaki [14].

(a) For a string $m$, we construct the generalized excursion measure $\boldsymbol{n}_{m}$ for the $\mathcal{L}_{m^{-}}$ diffusion process, where $\mathcal{L}_{m}=\frac{\mathrm{d}}{\mathrm{d} m} \frac{\mathrm{d}}{\mathrm{d} x}$. Let $\boldsymbol{n}_{\mathrm{BE}}$ denote Itô's Brownian excursion measure and let $\ell(t, x)$ denote the local time at $x \in(0, \infty)$ of the excursion path $(e(t): t \geq 0)$ under $\boldsymbol{n}_{\mathrm{BE}}$. Define $A_{m}(t)=\int_{(0, \infty)} \ell(t, x) \mathrm{d} m(x)$. We will show in Lemma 2.4 that $A_{m}(t)$ is finite or infinite $\boldsymbol{n}_{\mathrm{BE}}$-almost everywhere, according as $\int_{0+} x \mathrm{~d} m(x)$ is finite or infinite. If $\int_{0+} x \mathrm{~d} m(x)<\infty$, then we can consider the time-changed excursion path $e_{m}(\cdot)=e\left(A_{m}^{-1}(\cdot)\right)$ to obtain the desired measure by setting $\boldsymbol{n}_{m}(e \in \cdot)=\boldsymbol{n}_{\mathrm{BE}}\left(e_{m} \in \cdot\right)$ (Theorem 2.5).

We introduce the following condition, which we will call the $\mathcal{M}_{L}$-tightness condition:

$$
\lim _{\delta \rightarrow 0+} \limsup _{\lambda \rightarrow \infty} \int_{(0, \delta]} x \log \log (1 / x) \mathrm{d} m_{\lambda}(x)=0 .
$$

\footnotetext{
*E-mail: yano@kurims.kyoto-u.ac.jp
} 
Suppose that $m_{\lambda}(x) \rightarrow m(x)$ for each continuity point $x$ of $m$ and that the $\mathcal{M}_{L}$-tightness condition is satisfied. Then our Theorem 2.9] asserts that the excursion measure for $\mathcal{L}_{m_{\lambda}}$ converges to that for $\mathcal{L}_{m}$ in the sense that the time-changed excursion paths under $\boldsymbol{n}_{\mathrm{BE}}$ converge uniformly:

$$
\lim _{\lambda \rightarrow \infty} \sup _{t \geq 0}\left|e_{m_{\lambda}}(t)-e_{m}(t)\right|=0, \quad \boldsymbol{n}_{\mathrm{BE}} \text {-a.e. }
$$

We will see in Proposition 2.10 that the following $\mathcal{M}$-tightness condition

$$
\lim _{\delta \rightarrow 0+} \limsup _{\lambda \rightarrow \infty} \int_{(0, \delta]} x \mathrm{~d} m_{\lambda}(x)=0
$$

is close to necessary for the convergence (1.2).

(b) Iglehart [6] and Bolthausen [5] have proved that a suitably rescaled random walk on $\mathbb{R}$, conditioned to be positive until a fixed time, converges in law to the Brownian meander. Li-Shiga-Tomisaki [14] have generalized these results to prove that a suitably rescaled process for null recurrent generalized diffusion processes converges in law to the Bessel meander of dimension $d \in(0,2)$.

We generalize the conditional limit theorem of Li-Shiga-Tomisaki for positive recurrent diffusion processes. Let $m$ be a string on $(0, \infty)$. Following Kasahara-Watanabe [12, we suppose that $m(\infty)<\infty$ and that the difference $m(\infty)-m(x)$ is regularly varying as $x \rightarrow \infty$. Then a suitably rescaled process $e^{\lambda, u(\lambda)}(t):=e(\lambda t) / u(\lambda)$, conditioned to be positive until time one, namely

$$
\left(e^{\lambda, u(\lambda)}(t): t \in[0,1]\right) \text { under } \boldsymbol{Q}_{m}^{x}\left(\cdot \mid \zeta\left(e^{\lambda, u(\lambda)}\right)>1\right)
$$

converges in law to a Bessel meander on $[0,1]$ of negative dimension. See Section 2.3 for details.

(c) We must remark that the importance of certain tightness-like conditions in the class of strings was first pointed out by Kotani [13] and Kasahara-Watanabe [12.

We denote by $\mathcal{M}_{0}$ the class of strings $m$ for which 0 is both exit and entrance for $\mathcal{L}_{m}$. For a family of strings $\left\{m_{\lambda}\right\}$ in the class $\mathcal{M}_{0}$ such that $m_{\lambda}(0+) \geq 0$ for all $\lambda$, the pointwise convergence condition

$$
m_{\lambda}(x) \rightarrow m(x) \quad \text { as } \lambda \rightarrow \infty \text {, for each continuity point } x \text { of } m,
$$

plays an important role in various limit theorems for null recurrent diffusion processes. Moreover, this condition is also essential; In fact, Kasahara [11] has proved the bicontinuity of Krein's correspondence where the class $\mathcal{M}_{0}$ is equipped with a topology induced by the pointwise convergence (1.5). Based on this theory Kasahara [1] and Watanabe 20] have established the converse of limit theorems.

A major breakthrough both in the limit theory and in the spectral theory has recently been achieved by Kotani [13] and Kasahara-Watanabe [12. Let $\mathcal{M}_{1}$ denote the class of 
strings $m$ for which the origin is of limit circle type for $\mathcal{L}_{m}$. They have introduced the following condition for strings $m_{\lambda} \in \mathcal{M}_{1}$ :

$$
\lim _{\delta \rightarrow 0+} \limsup _{\lambda \rightarrow \infty} \int_{0}^{\delta} m_{\lambda}(x)^{2} \mathrm{~d} x=0 .
$$

This condition seems to mean a kind of tightness for the family of Radon measures $\mathrm{d} m_{\lambda}(x)$. For this reason we call the condition (1.6) the $\mathcal{M}_{1}$-tightness condition. We say that $m_{\lambda} \rightarrow m$ in $\mathcal{M}_{1}$ if both the pointwise convergence condition (1.5) and the $\mathcal{M}_{1^{-}}$ tightness condition (1.6) hold. Kotani has generalized Kasahara's continuity theorem for Krein's correspondence to the class $\mathcal{M}_{1}$. Kasahara-Watanabe have obtained limit theorems for the fluctuation of the occupation time and the inverse local time of positive recurrent diffusion processes.

(d) Let us recall the usual excursion theory. Consider a diffusion process on $[0, \infty)$ for which the origin is a reflecting boundary. Note that the origin is necessarily both exit and entrance in Feller's sense. Then one can construct the excursion point process by using the zero set of a sample path to cut the path into excursions. Itô [8] has shown that the point process is stationary Poisson whose characteristic measure is given by a $\sigma$-finite measure, which we call the Itô excursion measure. Conversely, one can construct a diffusion process from an Itô excursion measure by stringing together the excursions of the associated point process.

The Itô excursion measures have several descriptions. We list the following four formulae for the Brownian excursion measure $\boldsymbol{n}_{\mathrm{BE}}$. See the textbooks [7, [18] and [4] for details.

Let $M$ stand for the maximum value of the path. Let $\boldsymbol{Q}_{\mathrm{BM}}^{x}$ for $x>0$ stand for the law of the one-dimensional Brownian motion (with generator $\frac{1}{2} \frac{\mathrm{d}^{2}}{\mathrm{~d} x^{2}}$ ) starting from $x$ and absorbed at the origin. Let $\boldsymbol{P}_{3 \mathrm{~B}}^{x}$ for $x \geq 0$ stand for the $h$-transform of $\boldsymbol{Q}_{\mathrm{BM}}^{x}$ with respect to the Brownian scale, which is actually the law of the 3-dimensional Bessel process (with generator $\left.\frac{1}{2} \frac{\mathrm{d}^{2}}{\mathrm{~d} x^{2}}+\frac{1}{x} \frac{\mathrm{d}}{\mathrm{d} x}\right)$ starting from $x$.

(i) $\boldsymbol{n}_{\mathrm{BE}}(M=0)=0$ and for each $x>0$ and every bounded continuous functional $F$ on the excursion space whose support is contained in $\{M>x\}$ for some $x>0$,

$$
\boldsymbol{n}_{\mathrm{BE}}[F]=\lim _{a \rightarrow 0+} \frac{1}{a} \boldsymbol{Q}_{\mathrm{BM}}^{a}[F] .
$$

(ii) The strong Markov property: Under $\boldsymbol{n}_{\mathrm{BE}}$ the excursion process $(e(t): t \geq 0)$ is a strong Markov process with transition kernel $\boldsymbol{Q}_{\mathrm{BM}}^{x}(e(t) \in \mathrm{d} y)$ and entrance law $\frac{1}{x} \boldsymbol{P}_{3 \mathrm{~B}}^{0}(e(t) \in \mathrm{d} x)$. In particular, for each positive stopping time $\tau$ and every measurable set $\Gamma$,

$$
\boldsymbol{n}_{\mathrm{BE}}(e(\tau+\cdot) \in \Gamma)=\int_{(0, \infty)} \frac{1}{x} \boldsymbol{P}_{3 \mathrm{~B}}^{0}(e(\tau) \in \mathrm{d} x) \boldsymbol{Q}_{\mathrm{BM}}^{x}(\Gamma) .
$$

(iii) The maximum decomposition due to Williams [21]: For any measurable set $\Gamma$,

$$
\boldsymbol{n}_{\mathrm{BE}}(\Gamma)=\int_{0}^{\infty} \boldsymbol{R}_{3 \mathrm{~B}}^{x}(\Gamma) \frac{\mathrm{d} x}{x^{2}}
$$


Here $\boldsymbol{R}_{3 \mathrm{~B}}^{x}$ stands for the law of the path defined by piecing together two independent $\boldsymbol{P}_{3 \mathrm{~B}}^{x}$-processes (the second run backward in time) until they first hit the point $x$. The formula (1.9) means that the law of the maximum $M$ is given as $\boldsymbol{n}_{\mathrm{BE}}(M \in \mathrm{d} x)=\frac{\mathrm{d} x}{x^{2}}$ and the conditional law of $\boldsymbol{n}_{\mathrm{BE}}$ given $M=x$ is $\boldsymbol{R}_{3 \mathrm{~B}}^{x}$.

(iv) The lifetime decomposition: For any measurable set $\Gamma$,

$$
\boldsymbol{n}_{\mathrm{BE}}(\Gamma)=\int_{0}^{\infty} \boldsymbol{P}_{3 \mathrm{~B}}^{0}(\Gamma \mid e(t)=0) p_{3 \mathrm{~B}}(t, 0,0) \mathrm{d} t .
$$

Here $p_{3 \mathrm{~B}}(t, x, y)$ stands for the transition probability density of $\boldsymbol{P}_{3 B}^{x}$ with respect to its speed measure. The formula (1.10) means that the law of the lifetime $\zeta$ is given as $\boldsymbol{n}_{\mathrm{BE}}(\zeta \in \mathrm{d} t)=p_{3 \mathrm{~B}}(t, 0,0) \mathrm{d} t$ and the conditional law of $\boldsymbol{n}_{\mathrm{BE}}$ given $\zeta=t$ is $\boldsymbol{P}_{3 \mathrm{~B}}^{0}(\cdot \mid e(t)=0)$.

(e) Let $m$ be a string on $(0, \infty)$ such that the origin is exit and non-entrance for $\mathcal{L}_{m}$. Then there is no reflecting $\mathcal{L}_{m}$-diffusion process and therefore the usual excursion theory is not available. Nevertheless one can study a $\sigma$-finite measure on the excursion space such that the description formulae listed above still holds where we replace $\boldsymbol{Q}_{\mathrm{BM}}^{x}$ by the absorbing $\mathcal{L}_{m}$-diffusion process and $\boldsymbol{P}_{3 \mathrm{~B}}^{x}$ by its $h$-transform, etc. We call such a measure the generalized excursion measure for the $\mathcal{L}_{m}$-diffusion process.

Pitman-Yor [15] have introduced such generalized excursion measures and established the description formulae (i), (ii) and (iii). In [16] they established the formula (iv) for the Bessel processes of dimension $-\infty<d<2$. They used the generalized excursion measures for Bessel processes to obtain several remarkable path decomposition formulae for Bessel processes and Bessel bridges.

The use of time change, in the context of Itô's Brownian excursion measure, can be found in Biane-Yor [2, Section 3 (See also [1]). Their motivation was to compute the joint law of $(H(\eta(t)), \eta(t))$ where $H(t)$ denotes Cauchy's principal value of Brownian motion and $\eta(t)$ denotes the inverse local time at the origin.

The second author [23] has established the lifetime decomposition formula (iv) for generalized excursion measures assuming that the Laplace transform of the absorbing spectral measure, which is the counterpart of $p_{3 \mathrm{~B}}(t, 0,0)$ in (1.10), is finite. He also studied the relationship between the absorbing spectral measure and the (reflecting) spectral measure corresponding to dual strings to study Kasahara-Watanabe's limit theorem from this viewpoint. We do not go into the lifetime decomposition formula in the present paper.

The paper is organized as follows. In Section 2 we state our results. We will state the construction theorem of generalized excursion measures in Section 2.1 and the convergence theorem of generalized excursion measures in Section 2.2. Applications to limit theorems will be stated in Section 2.3. Sections 3, 4 and 5 are devoted to the proofs of the results given in Sections 2.1, 2.2 and 2.3, respectively. 


\section{Results}

Before stating our results we prepare some notation.

Let $E$ be the space of continuous paths $e:[0, \infty) \rightarrow[0, \infty)$ such that if $e\left(t_{0}\right)=0$ for some $t_{0}>0$ then $e(t)=0$ for all $t>t_{0}$. We set

$$
\zeta(e)=\inf \{t>0: e(t)=0\}
$$

where $\inf \emptyset=\infty$. We call $\zeta(e) \in[0, \infty]$ the lifetime of the path $e$. Hence, if $\zeta(e) \in(0, \infty)$, then $e(t)>0$ for $0<t<\zeta(e)$ and $e(t)=0$ for $t \geq \zeta(e)$. We regard $E$ as a complete separable metric space equipped with the compact uniform topology. Let $\mathcal{E}$ denote its Borel $\sigma$-field. Then all the measures $\boldsymbol{n}_{\mathrm{BE}}, \boldsymbol{P}_{3 \mathrm{~B}}^{x}$ for $x \geq 0, \boldsymbol{R}_{3 \mathrm{~B}}^{x}$ and $\boldsymbol{Q}_{\mathrm{BM}}^{x}$ for $x>0$ may be considered to be defined on $E$.

For $x \in(0, \infty)$, denote by $\tau_{x}$ the first passage time to $x$. Denote $M(e)=\max _{t \geq 0} e(t)$.

We fix versions of the local time at $x \in(0, \infty)$ for almost every path $e$ under $\boldsymbol{n}_{\mathrm{BE}}$, $\boldsymbol{P}_{3 \mathrm{~B}}^{x}$ for $x \geq 0, \boldsymbol{R}_{3 \mathrm{~B}}^{x}$ and $\boldsymbol{Q}_{\mathrm{BM}}^{x}$ for $x>0$. We will denote them by the common symbol $\ell(t, x)$. Thus, $\ell(t, x)$ is jointly continuous on $(0, \infty) \times(0, \infty)$ and the equality

$$
\int_{0}^{t} f(e(s)) \mathrm{d} s=2 \int_{(0, \infty)} f(x) \ell(t, x) \mathrm{d} x
$$

holds for every bounded Borel function $f$ on $(0, \infty)$ for almost every path $e$ with respect to the measure $\boldsymbol{n}_{\mathrm{BE}}$, and so on. For instance, we know by the the maximum decomposition formula (1.9) that the process $\left(\ell(t, \cdot): t \leq \tau_{x}\right)$ under $\boldsymbol{R}_{3 \mathrm{~B}}^{x}$ for $x>0$ has the same law as $\left(\ell(t, \cdot): t \leq \tau_{x}\right)$ under $\boldsymbol{P}_{3 \mathrm{~B}}^{0}$.

\section{Classes of strings}

A string $m$ on $(0, \infty)$ is a function $m:(0, \infty) \rightarrow(-\infty, \infty)$ which is strictly-increasing and right-continuous.

Remark 2.1. In the context of generalized diffusion processes, strings are only assumed to be non-decreasing and right-continuous.

We consider the following four classes of strings:

$$
\begin{aligned}
& \mathcal{M}_{0}=\{m: \text { string, } m(0+) \text { is finite }\} \\
& \mathcal{M}_{1}=\left\{m: \text { string, } \int_{0+} m(x)^{2} \mathrm{~d} x<\infty\right\} \\
& \mathcal{M}_{L}=\left\{m: \text { string, } \int_{0+} x \log \log (1 / x) \mathrm{d} m(x)<\infty\right\}
\end{aligned}
$$

and

$$
\mathcal{M}=\left\{m \text { : string, } \int_{0+} x \mathrm{~d} m(x)<\infty\right\} .
$$


Then the following relation holds:

$$
\mathcal{M}_{0} \subset \mathcal{M}_{1} \subset \mathcal{M}_{L} \subset \mathcal{M}
$$

The relation $\mathcal{M}_{1} \subset \mathcal{M}_{L}$ follows from the fact that there exists $C$ such that

$$
\int_{(0, \delta]} x \log \log (1 / x) \mathrm{d} m(x) \leq C\left(\int_{0}^{\delta} m(x)^{2} \mathrm{~d} x\right)^{1 / 2} \quad m \in \mathcal{M}_{1}, \delta<1 / 2 .
$$

In fact, we integrate the LHS by parts to obtain

$$
\int_{(0, \delta]} x \log \log (1 / x) \mathrm{d} m(x) \leq C^{\prime} \int_{0}^{\delta}|m(x)| \log \log (1 / x) \mathrm{d} x \quad m \in \mathcal{M}_{L}, \delta<1 / 2
$$

for some constant $C^{\prime}$.

Remark 2.2. (i) The origin is both exit and entrance for $\mathcal{L}_{m}$ if and only if $m \in \mathcal{M}_{0}$.

(ii) The origin is of limit circle type for $\mathcal{L}_{m}$ in Weyl's sense if and only if $m \in \mathcal{M}_{1}$.

(iii) The origin is exit for $\mathcal{L}_{m}$ if and only if $m \in \mathcal{M}$.

Example 2.3. For $\alpha \in(0, \infty)$, let

$$
m^{(\alpha)}(x)= \begin{cases}(1-\alpha)^{-1} x^{\frac{1}{\alpha}-1} & \text { if } \alpha \in(0,1), \\ \log x & \text { if } \alpha=1, \\ -(\alpha-1)^{-1} x^{\frac{1}{\alpha}-1} & \text { if } \alpha \in(1, \infty) .\end{cases}
$$

Note that $\mathrm{d} m^{(\alpha)}(x)=\alpha^{-1} x^{\frac{1}{\alpha}-2} \mathrm{~d} x$ for all $\alpha \in(0, \infty)$. Then

(i) $m^{(\alpha)} \in \mathcal{M}_{0}$ if and only if $\alpha \in(0,1)$;

(ii) $m^{(\alpha)} \in \mathcal{M}_{1}$ if and only if $\alpha \in(0,2)$;

(iii) $m^{(\alpha)} \in \mathcal{M}_{L}$ for all $\alpha \in(0, \infty)$.

\subsection{Construction of generalized excursion measures}

For a string $m$ on $(0, \infty)$, we define

$$
A_{m}(t)=\int_{(0, \infty)} \ell(t, x) \mathrm{d} m(x) \quad \text { for } t \geq 0 .
$$

Lemma 2.4. Let $m$ be a string on $(0, \infty)$. Then the following dichotomy holds:

(i) If $m \in \mathcal{M}$, then $A_{m}(t)<\infty$ for all $t \geq 0$, $\boldsymbol{n}_{\mathrm{BE}}-$ a.e.

(ii) If $m \notin \mathcal{M}$, then $A_{m}(t)=\infty$ for all $t \geq 0, \boldsymbol{n}_{\mathrm{BE}}-$ a.e.

In what follows we assume that $m \in \mathcal{M}$. It is easy to see that the function $A_{m}$ is continuous and strictly increasing on $(0, \zeta]$ and is constant on $[\zeta, \infty)$. Define $A_{m}^{-1}(t)$ for $t<A_{m}(\zeta)$ by the inverse function of $A_{m}$ and set $A_{m}^{-1}(t)=\zeta$ for $t \geq A_{m}(\zeta)$. Then $A_{m}^{-1}\left(A_{m}(t)\right)=t \wedge \zeta$ for all $t \geq 0$. We define the time-changed process on the space $E$ by

$$
e_{m}(t)=e\left(A_{m}^{-1}(t)\right) \quad t \geq 0
$$


and define a $\sigma$-finite measure on $E$ by

$$
\boldsymbol{n}_{m}(\cdot)=\boldsymbol{n}_{\mathrm{BE}}\left(e_{m} \in \cdot\right) .
$$

For $x>0$ let $\boldsymbol{Q}_{m}^{x}$ denote the law of the $\mathcal{L}_{m}$-diffusion process starting from $x$ and absorbed at the origin. Let $\boldsymbol{P}_{m}^{x}$ for $x \geq 0$ denote the $h$-transform of $\boldsymbol{Q}_{m}^{x}$ with respect to $h(x)=x$ (the Brownian scale function). This is actually the law of the diffusion process (starting from $x$ ) with speed measure $x^{2} \mathrm{~d} m(x)$ and scale function $-1 / x$. Let $\boldsymbol{R}_{m}^{x}$ for $x>0$ denote the law of the path defined by piecing together two independent $\boldsymbol{P}_{m}^{x}$-processes until they first hit the point $x$ (the second one being run backward in time).

Then we obtain the following description formulae for $\boldsymbol{n}_{m}$.

Theorem 2.5. Let $m \in \mathcal{M}$. Then the following hold:

$(\mathrm{i})^{\prime} \boldsymbol{n}_{m}(M=0)=0$ and for every bounded continuous functional $F$ on $E$ whose support is contained in $\{M>x\}$ for some $x>0$,

$$
\boldsymbol{n}_{m}[F]=\lim _{a \rightarrow 0+} \frac{1}{a} \boldsymbol{Q}_{m}^{a}[F] .
$$

(ii)' For each positive stopping time $\tau$ and every measurable set $\Gamma$,

$$
\boldsymbol{n}_{m}(e(\tau+\cdot) \in \Gamma)=\int_{(0, \infty)} \frac{1}{x} \boldsymbol{P}_{m}^{0}(e(\tau) \in \mathrm{d} x) \boldsymbol{Q}_{m}^{x}(\Gamma) .
$$

(iii)' For any measurable set $\Gamma$,

$$
\boldsymbol{n}_{m}(\Gamma)=\int_{0}^{\infty} \boldsymbol{R}_{m}^{x}(\Gamma) \frac{\mathrm{d} x}{x^{2}}
$$

For later use we note that if we put $\tau=\tau_{x}$ (for some fixed $x>0$ ) then the formula (ii)' becomes

$$
\boldsymbol{Q}_{m}^{x}(\Gamma)=x \boldsymbol{n}_{m}\left(e\left(\tau_{x}+\cdot\right) \in \Gamma\right)
$$

\subsection{Convergence theorem of generalized excursion measures}

Let $\left\{m_{\lambda}\right\}$ be a family of strings on $(0, \infty)$. As we have mentioned in the introduction, the pointwise convergence condition

$$
m_{\lambda}(x) \rightarrow m(x) \text { as } \lambda \rightarrow \infty \text { for all continuity points } x \text { of } m,
$$

is inadequate for the studies of various limit theorems and of the spectral theory.

In the class $\mathcal{M}_{0}$, it is usually assumed that $m_{\lambda}(0+) \geq 0$. We may regard it as the tightness condition of the class $\mathcal{M}_{0}$.

For the class $\mathcal{M}_{1}$, Kotani and Kasahara-Watanabe have introduced the condition

$$
\lim _{\delta \rightarrow 0+} \limsup _{\lambda \rightarrow \infty} \int_{0}^{\delta} m_{\lambda}(x)^{2} \mathrm{~d} x=0,
$$

which we call the $\mathcal{M}_{1}$-tightness condition.

For the classes $\mathcal{M}_{L}$ and $\mathcal{M}$, we consider the following tightness-like conditions. 
Definition 2.6. Let $m_{\lambda}, m \in \mathcal{M}$.

(i) The condition

$$
\lim _{\delta \rightarrow 0+} \limsup _{\lambda \rightarrow \infty} \int_{(0, \delta]} x \log \log (1 / x) \mathrm{d} m_{\lambda}(x)=0
$$

is called the $\mathcal{M}_{L}$-tightness condition.

(ii) The condition

$$
\lim _{\delta \rightarrow 0+} \limsup _{\lambda \rightarrow \infty} \int_{(0, \delta]} x \mathrm{~d} m_{\lambda}(x)=0
$$

is called the $\mathcal{M}$-tightness condition.

Definition 2.7. Let $m_{\lambda}, m \in \mathcal{M}$. We say that $m_{\lambda} \rightarrow m$ in $\mathcal{M}_{L}$ (resp. $\left.\mathcal{M}\right)$ if both the pointwise convergence condition (2.18) and the $\mathcal{M}_{L^{-}}$(resp. $\mathcal{M}-$ ) tightness condition hold.

Remark 2.8. It is immediate by (2.8) that $\mathcal{M}_{1}$-tightness implies $\mathcal{M}_{L}$-tightness. It is obvious by definition that $\mathcal{M}_{L}$-tightness implies $\mathcal{M}$-tightness.

The following theorem asserts that $\mathcal{M}_{L^{-}}$convergence implies pathwise uniform convergence of time-changed excursion processes.

Theorem 2.9 (Convergence theorem of generalized excursion measures). Assume that $m_{\lambda} \rightarrow m$ in $\mathcal{M}_{L}$ as $\lambda \rightarrow \infty$. Then

$$
\lim _{\lambda \rightarrow \infty} \sup _{t \geq 0}\left|A_{m_{\lambda}}(t)-A_{m}(t)\right|=0 \quad \boldsymbol{n}_{\mathrm{BE}}-\text { a.e. }
$$

and

$$
\lim _{\lambda \rightarrow \infty} \sup _{t \geq 0}\left|e_{m_{\lambda}}(t)-e_{m}(t)\right|=0 \quad \boldsymbol{n}_{\mathrm{BE}} \text {-a.e. }
$$

The following proposition asserts that the $\mathcal{M}$-tightness condition is necessary for the convergence (2.22).

Proposition 2.10. Let $m_{\lambda}, m \in \mathcal{M}$. Assume that the convergence (2.22) holds. Then the $\mathcal{M}$-tightness condition (2.21) holds.

\subsection{Conditional limit theorem}

For two functions $f$ and $g$ defined for all large reals, we write $f(x) \sim g(x)$ as $x \rightarrow \infty$ to mean that $\lim _{x \rightarrow \infty} f(x) / g(x)=1$. A function $K$ defined for large (real) $x$ is slowly varying as $x \rightarrow \infty$ provided $K(\lambda x) \sim K(\lambda)$ as $\lambda \rightarrow \infty$ for all sufficiently large $x$.

For $e \in E, \lambda_{1}>0$ and $\lambda_{2}>0$, we use the notation

$$
e^{\lambda_{1}, \lambda_{2}}(t)=\frac{1}{\lambda_{2}} e\left(\lambda_{1} t\right) \quad \text { for } t \geq 0
$$

First of all, we restate the conditional limit theorem of Li-Shiga-Tomisaki, using our terminology. Consider the following assumption: 
(A1) $\alpha \in(0,1), m \in \mathcal{M}_{0}$ and

$$
m(x) \sim(1-\alpha)^{-1} x^{\frac{1}{\alpha}-1} K(x) \quad \text { as } x \rightarrow \infty,
$$

for some function $K$ slowly varying at infinity.

Remark 2.11. Note that, if the assumption (A1) is satisfied, then the corresponding $\mathcal{L}_{m}$-diffusion process is null recurrent; in fact, $m(\infty)=\infty$.

Let $u(\lambda)$ be an asymptotic inverse of $\lambda^{\frac{1}{\alpha}} K(\lambda)$.

Theorem 2.12 (Li-Shiga-Tomisaki [14, Theorem 3.1]). Suppose that (A1) is satisfied. Then, for any $x>0$, the distribution on $E$ of the rescaled process $\left(e^{\lambda, u(\lambda)}(t): t \in\right.$ $(0,1])$ under $\boldsymbol{Q}_{m}^{x}\left(\cdot \mid \zeta\left(e^{\lambda, u(\lambda)}\right)>1\right)$ converges as $\lambda \rightarrow \infty$ to the process $(e(t): t \in(0,1])$ under $\boldsymbol{n}_{m^{(\alpha)}}(\cdot \mid \zeta>1)$ where $m^{(\alpha)} \in \mathcal{M}_{0}$ has been introduced in (2.10).

Remark 2.13. For $\alpha \in(0, \infty)$, the process $(e(t): t \in[0,1])$ under $\boldsymbol{n}_{m^{(\alpha)}}(\cdot \mid \zeta>1)$ is called the Bessel meander of dimension $d=2-2 \alpha$. Theorem 2.12 says that the limit process is a Bessel meander of positive dimension.

Remark 2.14. More precisely, Theorem 3.1 of [14] covers the case where a string is nondecreasing but is not strictly increasing so that the $\mathcal{L}_{m}$-diffusion process is a generalized diffusion process.

We introduce the following assumptions on a string $m \in \mathcal{M}$ :

(A2) $\alpha=1, m \in \mathcal{M}_{0}$ and

$$
m(\lambda x)-m(\lambda) \sim(\log x) K(\lambda) \quad \text { as } \lambda \rightarrow \infty, \text { for all } x>0,
$$

for some function $K$ slowly varying at infinity.

(A3) $\alpha \in(1, \infty)$. For small $x$, the string $m$ satisfies

$$
\lim _{x \rightarrow 0+} \frac{m(\infty)-m(x)}{x^{\frac{1}{\alpha}-1}}<\infty .
$$

For large $x$ it satisfies $m(\infty)<\infty$ and

$$
m(\infty)-m(x) \sim(\alpha-1)^{-1} x^{\frac{1}{\alpha}-1} K(x) \quad \text { as } x \rightarrow \infty,
$$

for some function $K$ slowly varying at infinity.

Remark 2.15. Note that the assumption (A3) implies that the corresponding $\mathcal{L}_{m^{-}}$ diffusion process is positive recurrent; In fact, $m(\infty)<\infty$.

Now let us state our generalization of Theorem 2.12, Let $u$ be an asymptotic inverse of $\lambda \mapsto \lambda^{\frac{1}{\alpha}} K(\lambda)$. 
Theorem 2.16 (Conditional limit theorem). Suppose that any one of (A1), (A2) and (A3) is satisfied. Then, for each $x>0$ and every bounded continuous functional $F$ on $E$,

$$
\boldsymbol{Q}_{m}^{x}\left[F\left(e^{\lambda, u(\lambda)}\right) \mid \zeta\left(e^{\lambda, u(\lambda)}\right)>1\right] \rightarrow \boldsymbol{n}_{m^{(\alpha)}}[F \mid \zeta>1] \quad \text { as } \lambda \rightarrow \infty
$$

where $m^{(\alpha)} \in \mathcal{M}$ has been introduced in (2.10) and where $e^{\lambda, u(\lambda)}(t)=e(\lambda t) / u(\lambda)$ as is defined in (2.24).

Theorem [2.16] says that in positive recurrent cases the limit process is the Bessel meander of the appropriate negative dimension.

The keys to the proof of Theorem 2.16] are the following three lemmas.

Lemma 2.17. Suppose that any one of (A1), (A2) and (A3) is satisfied. For $\lambda>0$, set

$$
m_{\lambda}(x)= \begin{cases}m(\lambda x) /\left\{\lambda^{\frac{1}{\alpha}-1} K(\lambda)\right\} & \text { if } \alpha \in(0,1), \\ \{m(\lambda x)-m(\lambda)\} / K(\lambda) & \text { if } \alpha=1, \\ \{m(\lambda x)-m(\infty)\} /\left\{\lambda^{\frac{1}{\alpha}-1} K(\lambda)\right\} & \text { if } \alpha \in(1, \infty) .\end{cases}
$$

so that $\mathrm{d} m_{\lambda}(x)=\mathrm{d} m(\lambda x) /\left\{\lambda^{\frac{1}{\alpha}-1} K(\lambda)\right\}$ in all cases. Then there exist constants $C, \lambda_{0}$ and $0<\varepsilon<1$ such that

$$
\left|m_{\lambda}(x)\right| \leq C x^{\varepsilon-1} \quad \text { for all } x \in(0,1] \text { and for all } \lambda>\lambda_{0} .
$$

In particular, $m_{\lambda} \rightarrow m^{(\alpha)}$ in $\mathcal{M}_{L}$ as $\lambda \rightarrow \infty$.

Lemma 2.18. Let $m \in \mathcal{M}$ and suppose that $\mathrm{d} m_{\lambda}(x)=\lambda \mathrm{d} m(\lambda x) / v(\lambda)$ Then, for each $x>0$ and every bounded continuous functional $F$ on $E$,

$$
\begin{aligned}
\boldsymbol{Q}_{m}^{x}[ & \left.F\left(e^{v(\lambda), \lambda}\right) \mid \zeta\left(e^{v(\lambda), \lambda}\right)>1\right] \\
& =\boldsymbol{n}_{\mathrm{BE}}\left[F\left(e\left(A_{m_{\lambda}}^{-1}\left(t+A_{m_{\lambda}}\left(\tau_{x / \lambda}\right)\right)\right): t \geq 0\right) \mid A_{m_{\lambda}}(\zeta)-A_{m_{\lambda}}\left(\tau_{x / \lambda}\right)>1\right] .
\end{aligned}
$$

Here $e^{v(\lambda), \lambda}(t)=\lambda^{-1} e(v(\lambda) t)$ as is defined in (2.24).

Lemma 2.19. Suppose that $m_{\lambda} \rightarrow m$ in $\mathcal{M}_{L}$ as $\lambda \rightarrow \infty$. Suppose, in addition, that $\boldsymbol{n}_{\mathrm{BE}}\left(A_{m}(\zeta)=1\right)=0$ and that the inequality (2.31) is satisfied for some constants $C, \lambda_{0}$ and $0<\varepsilon<1$. Then, for each $x>0$ and every bounded continuous functional $F$ on $E$,

$$
\begin{aligned}
\boldsymbol{n}_{\mathrm{BE}}\left[F\left(e_{m_{\lambda}}\right) ; A_{m_{\lambda}}(\zeta)-A_{m_{\lambda}}\left(\tau_{x / \lambda}\right)>1\right] & \\
\rightarrow & \rightarrow \boldsymbol{n}_{\mathrm{BE}}\left[F\left(e_{m}\right) ; A_{m}(\zeta)>1\right] \quad \text { as } \lambda \rightarrow \infty
\end{aligned}
$$

We point out that $\boldsymbol{n}_{\mathrm{BE}}$ is not a finite measure and hence that the bounded convergence theorem does not apply. 


\section{Proof of the construction theorem}

We begin with the proof of Lemma 2.4

Proof of Lemma 2.4. We want to show that $\boldsymbol{n}_{\mathrm{BE}}\left(A_{m}(t)=\infty, \exists t \geq 0\right)=0$ if $m \in \mathcal{M}$ and $\boldsymbol{n}_{\mathrm{BE}}\left(A_{m}(t)<\infty, \exists t \geq 0\right)=0$ if $m \notin \mathcal{M}$.

Since $A_{m}(t)$ is increasing on $[0, \zeta]$ and is constant on $[\zeta, \infty)$, we have

$$
\boldsymbol{n}_{\mathrm{BE}}\left(A_{m}(t)=\infty, \exists t \geq 0\right) \leq \boldsymbol{n}_{\mathrm{BE}}\left(A_{m}(\zeta)=\infty\right)
$$

Since the local time is an additive functional, we have $A_{m}(\zeta)=A_{m}\left(\tau_{M}\right)+A_{m}^{\vee}\left(\tau_{M}^{\vee}\right)$ where $A_{m}^{\vee}$ (resp. $\tau_{M}^{\vee}$ ) is the counterpart of $A_{m}$ (resp. $\left.\tau_{M}\right)$ for the time reversal path. Note that $\left\{A_{m}(\zeta)<\infty\right\}=\left\{A_{m}\left(\tau_{M}\right)<\infty\right\} \cap\left\{A_{m}^{\vee}\left(\tau_{M}^{\vee}\right)<\infty\right\}$. By definition of $\boldsymbol{R}_{3 \mathrm{~B}}^{a}$, the random variables $A_{m}\left(\tau_{M}\right)$ and $A_{m}^{\vee}\left(\tau_{M}^{\vee}\right)$ under $\boldsymbol{R}_{3 \mathrm{~B}}^{a}$ for $a>0$ are independent and both have the same distribution as $A_{m}\left(\tau_{a}\right)$ under $\boldsymbol{P}_{3 \mathrm{~B}}^{0}$. Hence we obtain

$$
\boldsymbol{n}_{\mathrm{BE}}\left(A_{m}(\zeta)=\infty\right)=\int_{0}^{\infty}\left\{1-\boldsymbol{P}_{3 \mathrm{~B}}^{0}\left(A_{m}\left(\tau_{a}\right)<\infty\right)^{2}\right\} \frac{\mathrm{d} a}{a^{2}} .
$$

Suppose that $A_{m}(t)<\infty$ for some $t \geq 0$. If $t \geq \tau_{M}$, then $A_{m}\left(\tau_{M}\right) \leq A_{m}(t)<\infty$. If $t<\tau_{M}$, then $\inf _{s \in\left[t, \tau_{M}\right]} e(s)>0$ and hence $A_{m}\left(\tau_{M}\right)=A_{m}\left(\tau_{M}\right)-A_{m}(t)+A_{m}(t)<\infty$. Thus

$$
\begin{aligned}
\boldsymbol{n}_{\mathrm{BE}}\left(A_{m}(t)<\infty, \exists t \geq 0\right) & \leq \boldsymbol{n}_{\mathrm{BE}}\left(A_{m}\left(\tau_{M}\right)<\infty\right) \\
& =\int_{0}^{\infty} \boldsymbol{P}_{3 \mathrm{~B}}^{0}\left(A_{m}\left(\tau_{a}\right)<\infty\right) \frac{\mathrm{d} a}{a^{2}} .
\end{aligned}
$$

The following 0-1 law is well-known (See, e.g. [22], Corollary 1): For any $a>0$,

$$
\boldsymbol{P}_{3 \mathrm{~B}}^{0}\left(A_{m}\left(\tau_{a}\right)<\infty\right)= \begin{cases}1 & \text { if } m \in \mathcal{M} \\ 0 & \text { if } m \notin \mathcal{M}\end{cases}
$$

Combining (3.1)-(3.4) with (3.5), we complete the proof.

Remark 3.1. The 0-1 law (3.5) is equivalent to

$$
\boldsymbol{P}_{3 \mathrm{~B}}^{0}\left(\int_{(0, a]} \ell(\infty, x) \mathrm{d} m(x)<\infty\right)= \begin{cases}1 & \text { if } m \in \mathcal{M} \\ 0 & \text { if } m \notin \mathcal{M}\end{cases}
$$

This is closely related to Jeulin's lemma ([10, Lemme (3.22)), a useful version of which can be found in Pitman-Yor [17, Lemma 2.

It is known that, if $m \in \mathcal{M}$, then $\boldsymbol{P}_{3 \mathrm{~B}}^{x}\left(A_{m}(t)<\infty, \forall t \geq 0\right)=1$ for $x \geq 0$ and $\boldsymbol{Q}_{\mathrm{BM}}^{x}\left(A_{m}(t)<\infty, \forall t \geq 0\right)=1$ for $x>0$. By a standard time-change argument (as in Chapter 5 of Itô-McKean's book [9]), we obtain the following. 
Proposition 3.2. (i) The time-changed process $e_{m}(t)=e\left(A_{m}^{-1}(t)\right)$ under $\boldsymbol{P}_{3 \mathrm{~B}}^{x}$ for $x \geq 0$ is a diffusion process whose law is $\boldsymbol{P}_{m}^{x}$ :

$$
\boldsymbol{P}_{3 \mathrm{~B}}^{x}\left(e_{m} \in \cdot\right)=\boldsymbol{P}_{m}^{x}(\cdot) \quad \text { for all } x \geq 0 .
$$

(ii) The time-changed process $e_{m}(t)=e\left(A_{m}^{-1}(t)\right)$ under $\boldsymbol{Q}_{\mathrm{BM}}^{x}$ for $x>0$ is a diffusion process whose law is $\boldsymbol{Q}_{m}^{x}$ :

$$
\boldsymbol{Q}_{\mathrm{BM}}^{x}\left(e_{m} \in \cdot\right)=\boldsymbol{Q}_{m}^{x}(\cdot) \quad \text { for all } x>0 .
$$

The proof is straightforward, so we omit it.

Proof of Theorem 2.5. Since $\tau=A_{m}^{-1}(t)$ is a positive stopping time, we can apply the description formula (1.8) to obtain

$$
\boldsymbol{n}_{\mathrm{BE}}\left(e\left(A_{m}^{-1}(t)+\cdot\right) \in \cdot\right)=\int_{(0, \infty)} \frac{1}{x} \boldsymbol{P}_{3 \mathrm{~B}}^{0}\left(A_{m}^{-1}(t) \in \mathrm{d} x\right) \boldsymbol{Q}_{\mathrm{BM}}^{x}(\cdot) .
$$

Consider the shifted path $e^{+}(s)=e\left(A_{m}^{-1}(t)+s\right), s \geq 0$. We denote the counterpart of $A_{m}$ for the shifted path $e^{+}$by $A_{m}^{+}$. Then we have $A_{m}^{+}(\cdot)=A_{m}\left(A_{m}^{-1}(t)+\cdot\right)-A_{m}\left(A_{m}^{-1}(t)\right)$ and then we have $\left(A_{m}^{+}\right)^{-1}(s)=A_{m}^{-1}(t+s)-A_{m}^{-1}(t)$. Hence by (3.9) we obtain

$$
\begin{aligned}
\boldsymbol{n}_{\mathrm{BE}}\left(e\left(A_{m}^{-1}(t+\cdot)\right) \in \Gamma\right) & =\boldsymbol{n}_{\mathrm{BE}}\left(e^{+}\left(\left(A_{m}^{+}\right)^{-1}(\cdot)\right) \in \Gamma\right) \\
& =\int_{(0, \infty)} \frac{1}{x} \boldsymbol{P}_{3 \mathrm{~B}}^{0}\left(A_{m}^{-1}(t) \in \mathrm{d} x\right) \boldsymbol{Q}_{\mathrm{BM}}^{x}\left(e\left(A_{m}^{-1}(\cdot)\right) \in \Gamma\right)
\end{aligned}
$$

for any measurable set $\Gamma$ of $E$. Therefore we obtain the formula (ii)' from Proposition 3.2 .

The formula (i) ${ }^{\prime}$ is an immediate consequence of (2.17), which is a special case of the formula (ii)'.

The maximum decomposition formula (iii)' is obvious from Proposition [3.2. In fact, the maximum value is invariant under time change, so $M\left(e_{m}\right)=M(e)$.

\section{Proof of the convergence theorem}

We remark the following two elementary facts without proofs.

Lemma 4.1. Let $f_{\lambda}$ and $f$ be non-decreasing functions on $[a, b]$ with $-\infty<a<b<\infty$. Suppose that $f$ is continuous and that $f_{\lambda}(x) \rightarrow f(x)$ for all $x \in[a, b]$. Then $f_{\lambda}(x) \rightarrow f(x)$ uniformly in $x \in[a, b]$.

Lemma 4.2. Let $f_{\lambda}$ and $f$ be non-decreasing functions on $[a, b]$ with $-\infty<a<b<\infty$. Suppose that $f$ is strictly increasing and that $f_{\lambda}(x) \rightarrow f(x)$ as $n \rightarrow \infty$ uniformly in $x \in[a, b]$. Then $f_{\lambda}^{-1}(x) \rightarrow f^{-1}(x)$ uniformly in $x \in(f(a), f(b))$.

Now we proceed to prove Theorem 2.9 
Proof of Theorem [2.9. $\mathbf{1}^{\circ}$ ). By the definition (2.11), it suffices to show that $A_{m_{\lambda}}(t) \rightarrow$ $A_{m}(t)$ as $\lambda \rightarrow \infty$ for any fixed $t>0$ and for $\boldsymbol{n}_{\mathrm{BE}}$-a.e. path $e$. Let $\delta>0$. Then

$$
\left|A_{m_{\lambda}}(t)-A_{m}(t)\right| \leq(\mathrm{I})+(\mathrm{II})
$$

where

$$
(\mathrm{I})=\left|\int_{(\delta, M(e)]} \ell(t, x) \mathrm{d} m_{\lambda}(x)-\int_{(\delta, M(e)]} \ell(t, x) \mathrm{d} m(x)\right|
$$

and

$$
(\mathrm{II})=\int_{(0, \delta]} \ell(\zeta, x)\left(\mathrm{d} m_{\lambda}(x)+\mathrm{d} m(x)\right) .
$$

$\left.2^{\circ}\right)$. By the pointwise convergence condition (2.18), we see that $\int_{(a, b]} f(x) \mathrm{d} m_{\lambda}(x) \rightarrow$ $\int_{(a, b]} f(x) \mathrm{d} m(x)$ as $\lambda \rightarrow \infty$ for all $0<a<b<\infty$ and each bounded continuous function $f$ on $[a, b]$. Therefore (I) converges to 0 as $\lambda \rightarrow \infty$.

$\left.3^{\circ}\right)$. Now to complete the proof it suffices to show that

$$
\lim _{\delta \rightarrow 0+} \limsup _{\lambda \rightarrow \infty}(\mathrm{II})=0 \quad \boldsymbol{n}_{\mathrm{BE}} \text {-a.e. }
$$

By an argument like that used in the proof of Lemma 2.4, we can reduce the convergence (4.4) to

$$
\lim _{\delta \rightarrow 0+} \limsup _{\lambda \rightarrow \infty} \int_{(0, \delta]} \ell(\infty, x)\left(\mathrm{d} m_{\lambda}(x)+\mathrm{d} m(x)\right)=0 \quad \boldsymbol{P}_{3 \mathrm{~B}^{-}}^{0} \text { a.e. }
$$

$\left.4^{\circ}\right)$. By the Ray-Knight theorem, we know that the process $(\ell(\infty, x): x \geq 0)$ under $\boldsymbol{P}_{3 \mathrm{~B}}^{0}$ obeys the law of the two-dimensional Bessel-squared process starting from the origin, which we denote by $\{(U(x): x \geq 0), \boldsymbol{U}\}$. Note that the transition kernel of this process is given by $q_{x}^{2}(a, b) \mathrm{d} b$ where

$$
q_{x}^{2}(a, b)=\frac{1}{2 x} \exp \left(-\frac{a+b}{2 x}\right) I_{0}\left(\frac{\sqrt{a b}}{x}\right), \quad a, b \geq 0 .
$$

The convergence (4.5) is equivalent to

$$
\lim _{\delta \rightarrow 0+} \limsup _{\lambda \rightarrow \infty} \int_{(0, \delta]} U(x)\left(\mathrm{d} m_{\lambda}(x)+\mathrm{d} m(x)\right)=0 \quad \boldsymbol{U} \text {-a.s. }
$$

By the law of the iterated logarithm, there exists a finite random variable $C$ such that $U(x) \leq C x \log \log (1 / x)$ for $x \in(0,1), \boldsymbol{U}$-a.s. Hence we obtain (4.7) by the assumption of the $\mathcal{M}_{L}$-tightness condition and the condition $m \in \mathcal{M}_{L}$. This completes the proof of (2.22).

$\left.5^{\circ}\right)$. Now we apply Lemma 4.2 to obtain

$$
\lim _{\lambda \rightarrow \infty} \sup _{t \geq 0}\left|A_{m_{\lambda}}^{-1}(t)-A_{m}^{-1}(t)\right|=0 \quad \boldsymbol{n}_{\mathrm{BE}} \text {-a.e. }
$$

Since $\boldsymbol{n}_{\mathrm{BE}}$-a.e. path $e$ is uniformly continuous, we obtain the desired convergence (2.23). 
Finally, we prove Proposition 2.10.

Proof of Proposition 2.10. We imitate the proof of Lemma 2 of [22. Assume that the convergence (2.22) holds. Let $\delta>0$ be fixed for a while. By the maximum decomposition formula (1.9), we have

$$
\lim _{\lambda \rightarrow \infty} \int_{(0, \delta]} \ell\left(\tau_{\delta}, x\right) \mathrm{d} m_{\lambda}(x)=\int_{(0, \delta]} \ell\left(\tau_{\delta}, x\right) \mathrm{d} m(x), \forall \delta>0 \quad \boldsymbol{P}_{3 \mathrm{~B}^{- \text {a.s. }}}^{0}
$$

By the Ray-Knight theorem, we know that the process $\left(\ell\left(\tau_{\delta}, x\right): x \in[0, \delta]\right)$ under $\boldsymbol{P}_{3 \mathrm{~B}}^{0}$ obeys the law of the 2-dimensional Bessel-squared process starting from the origin pinned at the origin when $x=\delta$, which we denote by $\left\{(U(x): x \in[0, \delta]), \boldsymbol{U}_{\delta}\right\}$. Note that

$$
\begin{aligned}
\boldsymbol{U}_{\delta}(U(x) \in \mathrm{d} a) & =\frac{q_{x}^{2}(0, a) q_{\delta-x}^{2}(a, 0)}{q_{\delta}^{2}(0,0)} \mathrm{d} a \\
& =\frac{\delta}{2 x(\delta-x)} \exp \left(-\frac{\delta a}{2 x(\delta-x)}\right) \mathrm{d} a
\end{aligned}
$$

where $q_{x}^{2}(a, b)$ is given in (4.6) . Then we have

$$
\boldsymbol{U}_{\delta}[U(x) / x]=\frac{2(\delta-x)}{\delta} \leq 2, \quad x \in(0, \delta]
$$

and

$$
\begin{gathered}
\boldsymbol{U}_{\delta}(U(x) / x \leq u)=1-\exp \left(-\frac{\delta u}{2(\delta-x)}\right), \quad x \in(0, \delta], u \in[0, \infty) \\
\leq 1-\mathrm{e}^{-u}, \quad x \in(0, \delta / 2], u \in[0, \infty) .
\end{gathered}
$$

By the convergence (4.9), we can take $\lambda_{\delta}>0$ so large that $\boldsymbol{U}_{\delta}\left(B_{\delta}\right) \geq 1 / 2$ where

$$
\begin{aligned}
B_{\delta}= & \left\{\lim _{\lambda \rightarrow \infty} \int_{(0, \delta]} U(x) \mathrm{d} m_{\lambda}(x)=\int_{(0, \delta]} U(x) \mathrm{d} m(x)\right\} \\
& \cap\left\{\sup _{\lambda>\lambda_{\delta}} \int_{(0, \delta]} U(x) \mathrm{d} m_{\lambda}(x) \leq L_{\delta}\right\}
\end{aligned}
$$

and

$$
L_{\delta}=\int_{(0, \delta]} U(x) \mathrm{d} m(x)+1
$$

In fact,

$$
\lim _{\lambda \rightarrow \infty} \boldsymbol{U}_{\delta}\left(\sup _{\lambda^{\prime}>\lambda}\left|\int_{(0, \delta]} U(x) \mathrm{d} m_{\lambda^{\prime}}(x)-\int_{(0, \delta]} U(x) \mathrm{d} m(x)\right|>1\right)=0 .
$$


By (4.12), we have $\boldsymbol{U}_{\delta}\left[L_{\delta}\right]<\infty$. We can now apply the dominated convergence theorem to obtain

$$
\begin{aligned}
\lim _{\lambda \rightarrow \infty} \boldsymbol{U}_{\delta}\left[1_{B} \int_{(0, \delta]} U(x) \mathrm{d} m_{\lambda}(x)\right] & =\boldsymbol{U}_{\delta}\left[1_{B} \int_{(0, \delta]} U(x) \mathrm{d} m(x)\right] \\
& \leq \int_{(0, \delta]} \boldsymbol{U}_{\delta}[U(x) / x] x \mathrm{~d} m(x) \\
& \leq 2 \int_{(0, \delta]} x \mathrm{~d} m(x) .
\end{aligned}
$$

On the other hand, we have

$$
\begin{aligned}
\boldsymbol{U}_{\delta}\left[1_{B} \int_{(0, \delta]} U(x) \mathrm{d} m_{\lambda}(x)\right] & =\int_{(0, \delta]} \boldsymbol{U}_{\delta}\left[1_{B} U(x) / x\right] x \mathrm{~d} m_{\lambda}(x) \\
& =\int_{(0, \delta]} x \mathrm{~d} m_{\lambda}(x) \int_{0}^{\infty} \boldsymbol{U}_{\delta}(B \cap\{U(x) / x>u\}) \mathrm{d} u
\end{aligned}
$$$$
\geq \int_{(0, \delta]} x \mathrm{~d} m_{\lambda}(x) \int_{0}^{\infty}\left[\boldsymbol{U}_{\delta}(B)-\boldsymbol{U}_{\delta}\{U(x) / x \leq u\}\right]^{+} \mathrm{d} u
$$$$
\geq C \int_{(0, \delta / 2]} x \mathrm{~d} m_{\lambda}(x)
$$

where $C=\int_{0}^{\infty}\left[\mathrm{e}^{-u}-1 / 2\right]^{+} \mathrm{d} u>0$. (Here we used (4.14).) Therefore we obtain

$$
\limsup _{\lambda \rightarrow \infty} \int_{(0, \delta / 2]} x \mathrm{~d} m_{\lambda}(x) \leq \frac{2}{C} \int_{(0, \delta]} x \mathrm{~d} m(x) .
$$

If we let $\delta$ tend to $0+$, then the RHS of (4.26) vanishes.

\section{Proof of the limit theorem}

Firstly, we prove Lemma 2.17

Proof of Lemma 2.17. $\mathbf{1}^{\circ}$ ). In the case of (A1), the inequality (2.31) is easily justified since $0<m_{\lambda}(x) \leq m_{\lambda}(1)$ for $x \in(0,1]$ and for $\lambda>0$ and $m_{\lambda}(1) \rightarrow 1$ as $\lambda \rightarrow \infty$.

$\left.\mathbf{2}^{\circ}\right)$. In the case of (A2), we may take $m(0):=m(0+)$ so that $m(x)$ is locally bounded on $[0, \infty)$. Hence we may apply Theorem 3.8.6 (b) of [3], pp.172, and then we obtain the inequality (2.31).

$\mathbf{3}^{\circ}$ ). In the case of (A3), using the two conditions (2.27) and (2.28), we may take a constant $C_{1}$ and a function $\widetilde{K}(x)$ defined on $[0, \infty)$ such that the following hold:

(i) $m(\infty)-m(x) \leq C_{1} x^{\frac{1}{\alpha}-1} \widetilde{K}(x)$ for all $x>0$.

(ii) $\widetilde{K}(x)$ is bounded away from 0 and $\infty$ on any compact subset of $[0, \infty)$.

(iii) $\widetilde{K}(x) / K(x) \rightarrow 1$ as $x \rightarrow \infty$. (Then $\widetilde{K}(x)$ is necessarily slowly varying as $x \rightarrow \infty$.) 
We may apply Theorem 1.5 .6 (ii) of [3], pp.25, to the function $\widetilde{K}(x)$, and see that there exist a constant $C$ such that

$$
\widetilde{K}(\lambda x) / \widetilde{K}(\lambda) \leq C_{2} x^{-\frac{1}{2 \alpha}-1}, \quad x \in(0,1], \lambda>0 .
$$

Now we obtain

$$
\left|m_{\lambda}(x)\right|=\frac{m(\infty)-m(\lambda x)}{\lambda^{\frac{1}{\alpha}-1} K(\lambda)} \leq C x^{\frac{1}{2 \alpha}-1} \quad x \in(0,1], \lambda>0
$$

for some constant $C$. Hence we obtain the inequality (2.31) with $\varepsilon=\frac{1}{2 \alpha}$.

$4^{\circ}$ ). Since $\int_{0}^{1 / 2} x^{\varepsilon-1} \log \log (1 / x) \mathrm{d} x<\infty$, we can apply the dominated convergence theorem to obtain

$$
\lim _{\lambda \rightarrow \infty} \int_{0}^{\delta}\left|m_{\lambda}(x)\right| \log \log (1 / x) \mathrm{d} x=\int_{0}^{\delta}\left|m^{(\alpha)}(x)\right| \log \log (1 / x) \mathrm{d} x, \quad \delta<1 / 2 .
$$

Therefore we conclude that the $\mathcal{M}_{L}$-tightness condition (2.20) holds by (2.9) and (5.3) . Since it is obvious that $m_{\lambda}(x) \rightarrow m^{(\alpha)}(x)$ pointwise, we conclude that $m_{\lambda} \rightarrow m$ in $\mathcal{M}_{L}$.

Secondly, we prove Lemma 2.18 .

Proof of Lemma 2.18. By (2.17), we have

$$
\boldsymbol{Q}_{m}^{x}\left[F\left(\lambda^{-1} e(v(\lambda) t): t \geq 0\right)\right]=x \boldsymbol{n}_{m}\left[F\left(\lambda^{-1} e\left(v(\lambda) t+\tau_{x}\right): t \geq 0\right)\right] .
$$

Since $\tau_{x}\left(e_{m}\right)=A_{m}\left(\tau_{x}\right)$ and $\zeta\left(e_{m}\right)=A_{m}(\zeta)$, the RHS is equal to

$$
x \boldsymbol{n}_{\mathrm{BE}}\left[F\left(\lambda^{-1} e\left(A_{m}^{-1}\left(v(\lambda) t+A_{m}\left(\tau_{x}\right)\right)\right): t \geq 0\right)\right] .
$$

Now consider $\widetilde{e}(t)=\lambda e\left(t / \lambda^{2}\right)$. By the scaling property of Bessel processes, we have $\boldsymbol{n}_{\mathrm{BE}}(\widetilde{e} \in)=\lambda \boldsymbol{n}_{\mathrm{BE}}(e \in \cdot)$. Then (5.5) can be rewritten as

$$
\frac{x}{\lambda} \boldsymbol{n}_{\mathrm{BE}}\left[F\left(\lambda^{-1} \widetilde{e}\left({\widetilde{A_{m}}}^{-1}\left(v(\lambda) t+\widetilde{A_{m}}\left(\widetilde{\tau_{x}}\right)\right)\right): t \geq 0\right)\right] .
$$

Here note that $\widetilde{\zeta}=\zeta(\widetilde{e})=\lambda^{2} \zeta$, that $\widetilde{\tau_{x}}=\tau_{x}(\widetilde{e})=\lambda^{2} \tau_{x / \lambda}$, and that $\widetilde{A_{m}}(t)=v(\lambda) A_{m_{\lambda}}\left(t / \lambda^{2}\right)$. Thus (5.6) leads to

$$
\frac{x}{\lambda} \boldsymbol{n}_{\mathrm{BE}}\left[F\left(e\left(A_{m}^{-1}\left(t+A_{m}\left(\tau_{x / \lambda}\right)\right)\right): t \geq 0\right)\right] .
$$

If we replace $F(e)$ by $F(e) 1_{\{\zeta(e)>1\}}$, then we obtain (2.32) from (5.4) and (5.7).

Thirdly, we prove Lemma 2.19 .

Proof of Lemma 2.19. $\left.\mathbf{1}^{\circ}\right)$. By Theorem 2.9, we have $F\left(e_{m_{\lambda}}\right) \rightarrow F\left(e_{m}\right)$ as $\lambda \rightarrow \infty$. By Theorem 2.9 again, we have $A_{m_{\lambda}}(\zeta)-A_{m_{\lambda}}\left(\tau_{x / \lambda}\right) \rightarrow A_{m}(\zeta) \boldsymbol{n}_{\mathrm{BE}}$-a.e. Since $\boldsymbol{n}_{\mathrm{BE}}\left(A_{m}(\zeta)=\right.$ 
1) $=0$, we see that the indicator function of $\left\{A_{m_{\lambda}}(\zeta)-A_{m_{\lambda}}\left(\tau_{x / \lambda}\right)>1\right\}$ converges to that of $\left\{A_{m}(\zeta)>1\right\} \boldsymbol{n}_{\mathrm{BE}^{-}}$a.e. Hence, for every $\delta>0$, we obtain

$$
\begin{gathered}
\lim _{\lambda \rightarrow \infty} \boldsymbol{n}_{\mathrm{BE}}\left[F\left(e_{m_{\lambda}}\right) ; A_{m_{\lambda}}(\zeta)-A_{m_{\lambda}}\left(\tau_{x / \lambda}\right)>1, M>\delta\right] \\
=\boldsymbol{n}_{\mathrm{BE}}\left[F\left(e_{m}\right) ; A_{m}(\zeta)>1, M>\delta\right]
\end{gathered}
$$

by the dominated convergence theorem.

$2^{\circ}$ ). Now it is sufficient to show that

$$
\lim _{\delta \rightarrow 0+} \sup _{\lambda>\lambda_{0}} \boldsymbol{n}_{\mathrm{BE}}\left[F\left(e_{m_{\lambda}}\right) ; A_{m_{\lambda}}(\zeta)-A_{m_{\lambda}}\left(\tau_{x / \lambda}\right)>1, M \leq \delta\right]=0 .
$$

Noting that $F$ is bounded and that $A_{m_{\lambda}}(\zeta)-A_{m_{\lambda}}\left(\tau_{x / \lambda}\right) \leq A_{m_{\lambda}}(\zeta)$, we have only to show that

$$
\lim _{\delta \rightarrow 0+} \sup _{\lambda>\lambda_{0}} \boldsymbol{n}_{\mathrm{BE}}\left(A_{m_{\lambda}}(\zeta)>1, M \leq \delta\right)=0 .
$$

Using the terminology in the proof of Lemma 2.4, we have $\left\{A_{m_{\lambda}}(\zeta)>1\right\} \subset\left\{A_{m_{\lambda}}\left(\tau_{a}\right)>\right.$ $1 / 2\} \cup\left\{A_{m_{\lambda}}^{\vee}\left(\tau_{a}^{\vee}\right)>1 / 2\right\}$, and hence we have

$$
\boldsymbol{n}_{\mathrm{BE}}\left(A_{m_{\lambda}}(\zeta)>1, M \leq \delta\right) \leq 2 \int_{0}^{\delta} \boldsymbol{P}_{3 \mathrm{~B}}^{0}\left(A_{m_{\lambda}}\left(\tau_{a}\right)>1 / 2\right) \frac{\mathrm{d} a}{a^{2}}
$$

by the maximum decomposition formula (11.9). Noting that $A_{m_{\lambda}}\left(\tau_{a}\right) \leq \int_{(0, a]} \ell(\infty, x) \mathrm{d} m_{\lambda}(x)$ and that $(\ell(\infty, x): x \geq 0)$ under $\boldsymbol{P}_{3 \mathrm{~B}}^{0}$ has the same law as the two-dimensional Besselsquared process, the RHS of (5.11) is dominated by

$$
2 \int_{0}^{\delta} \boldsymbol{P}\left(\int_{(0, a]} B(x)^{2} \mathrm{~d} m_{\lambda}(x)>1 / 4\right) \frac{\mathrm{d} a}{a^{2}}
$$

where $\{(B(x): x \geq 0), \boldsymbol{P}\}$ is the law of the standard Brownian motion. Hence it suffices to find a function $f(a)$ defined near $a=0$ with $\int_{0+} f(a) \frac{\mathrm{d} a}{a^{2}}<\infty$ such that $\sup _{\lambda>\lambda_{0}} \boldsymbol{P}\left(\int_{(0, a]} B(x)^{2} \mathrm{~d} m_{\lambda}(x)>1 / 4\right) \leq f(a)$ near $a=0$.

$\left.3^{\circ}\right)$. Now we proceed to find such a function $f(a)$. Set $m_{\lambda}^{a}(x)=a^{1-\varepsilon} m_{\lambda}(a x)$. Then

$$
\left|m_{\lambda}^{a}(x)\right| \leq C x^{\varepsilon-1} \quad \text { for all } x, a \in(0,1] \text { and } \lambda>\lambda_{0}
$$

by the assumption (2.31). By the scaling property of Brownian motion, the random variable $\int_{(0, a]} B(x)^{2} \mathrm{~d} m_{\lambda}(x)$ has the same law as $a^{\varepsilon} \int_{(0,1]} B(x)^{2} \mathrm{~d} m_{\lambda}^{a}(x)$ under $\boldsymbol{P}$. By Chebyshev's inequality, we have

$$
\boldsymbol{P}\left(a^{\varepsilon} \int_{(0,1]} B(x)^{2} \mathrm{~d} m_{\lambda}^{a}(x)>1 / 4\right) \leq \mathrm{e}^{-\rho a^{-\varepsilon} / 4} \boldsymbol{P}\left[\exp \left\{\rho \int_{(0,1]} B(x)^{2} \mathrm{~d} m_{\lambda}^{a}(x)\right\}\right]
$$

for all $a \in(0,1]$ and for all $\rho>0$. Hence it suffices to find $\rho>0$ such that

$$
C^{\prime}:=\sup _{\lambda>\lambda_{0}} \sup _{a \in(0,1]} \boldsymbol{P}\left[\exp \left\{\rho \int_{(0,1]} B(x)^{2} \mathrm{~d} m_{\lambda}^{a}(x)\right\}\right]<\infty .
$$


Indeed, we can choose $f(a)=C^{\prime} \mathrm{e}^{-\rho a^{-\varepsilon} / 4}$.

$\left.4^{\circ}\right)$. By Itô's formula, we see that

$$
\int_{(0,1]} B(x)^{2} \mathrm{~d} m_{\lambda}^{a}(x)=G_{\lambda}^{a}+H_{\lambda}^{a}+I_{\lambda}^{a}
$$

where $G_{\lambda}^{a}=B(1)^{2} m_{\lambda}^{a}(1), I_{\lambda}^{a}=-\int_{0}^{1} m_{\lambda}^{a}(x) B(x)^{2} \mathrm{~d} x$ and

$$
H_{\lambda}^{a}=-2 \int_{0}^{1} m_{\lambda}^{a}(x) B(x) \mathrm{d} B(x) .
$$

Here we remark that $\lim _{x \rightarrow 0+} B(x)^{2}\left|m_{\lambda}^{a}(x)\right|=0 \boldsymbol{P}$-a.s. by (5.13). Note that $G_{\lambda}^{a} \leq C B(1)^{2}$ and that $I_{\lambda}^{a} \leq C \int_{0}^{1} x^{\varepsilon-1} B(x)^{2} \mathrm{~d} x$. We also note that $M(x)=-2 \int_{0}^{x} m_{\lambda}^{a}(y) B(y) \mathrm{d} B(y)$ is a martingale such that

$$
\langle M\rangle(1)=4 \int_{0}^{1} m_{\lambda}^{a}(x)^{2} B(x)^{2} \mathrm{~d} x \leq 4 C^{2} \int_{0}^{1} x^{2 \varepsilon-2} B(x)^{2} \mathrm{~d} x .
$$

Since $B(1)^{2}, \int_{0}^{1} x^{\varepsilon-1} B(x)^{2} \mathrm{~d} x$ and $\int_{0}^{1} x^{2 \varepsilon-2} B(x)^{2} \mathrm{~d} x$ are all quadratic Wiener functionals, they are exponentially integrable if we take the exponent sufficiently small.

Proof of Theorem 2.16. Since the law of the lifetime of the Bessel meander is absolutely continuous, we have $\boldsymbol{n}_{\mathrm{BE}}\left(A_{m^{(\alpha)}}(\zeta)=1\right)=0$. Now we apply Lemmas 2.17, 2.18] and 2.19. putting $v(\lambda)=\lambda^{\frac{1}{\alpha}} K(\lambda)$, to obtain the desired conclusion.

\section{References}

[1] Ph. Biane and M. Yor. Valeurs principales associées aux temps locaux browniens et processus stables symétriques. C. R. Acad. Sci. Paris Sér. I Math., 300(20):695-698, 1985.

[2] Ph. Biane and M. Yor. Valeurs principales associées aux temps locaux browniens. Bull. Sci. Math. (2), 111(1):23-101, 1987.

[3] N. H. Bingham, C. M. Goldie, and J. L. Teugels. Regular variation, volume 27 of Encyclopedia of Mathematics and its Applications. Cambridge University Press, Cambridge, 1989.

[4] R. M. Blumenthal. Excursions of Markov processes. Probability and its Applications. Birkhäuser Boston Inc., Boston, MA, 1992.

[5] E. Bolthausen. On a functional central limit theorem for random walks conditioned to stay positive. Ann. Probability, 4(3):480-485, 1976.

[6] D. L. Iglehart. Functional central limit theorems for random walks conditioned to stay positive. Ann. Probability, 2:608-619, 1974. 
[7] N. Ikeda and S. Watanabe. Stochastic differential equations and diffusion processes, volume 24 of North-Holland Mathematical Library. North-Holland Publishing Co., Amsterdam, second edition, 1989.

[8] K. Itô. Poisson point processes attached to Markov processes. In Proceedings of the Sixth Berkeley Symposium on Mathematical Statistics and Probability (Univ. California, Berkeley, Calif., 1970/1971), Vol. III: Probability theory, pages 225-239, Berkeley, Calif., 1972. Univ. California Press.

[9] K. Itô and H. P. McKean, Jr. Diffusion processes and their sample paths. SpringerVerlag, Berlin, 1974. Second printing, corrected, Die Grundlehren der mathematischen Wissenschaften, Band 125.

[10] T. Jeulin. Semi-martingales et grossissement d'une filtration, volume 833 of Lecture Notes in Mathematics. Springer, Berlin, 1980.

[11] Y. Kasahara. Spectral theory of generalized second order differential operators and its applications to Markov processes. Japan. J. Math. (N.S.), 1(1):67-84, 1975/76.

[12] Y. Kasahara and S. Watanabe. Brownian representation of a class of Lévy processes and its application to occupation times of diffusion processes. Illinois J. Math., to appear.

[13] S. Kotani. Short Krein spaces. preprint.

[14] Z. Li, T. Shiga, and M. Tomisaki. A conditional limit theorem for generalized diffusion processes. J. Math. Kyoto Univ., 43(3):567-583, 2003.

[15] J. Pitman and M. Yor. A decomposition of Bessel bridges. Z. Wahrsch. Verw. Gebiete, 59(4):425-457, 1982.

[16] J. Pitman and M. Yor. Decomposition at the maximum for excursions and bridges of one-dimensional diffusions. In Itô's stochastic calculus and probability theory, pages 293-310. Springer, Tokyo, 1996.

[17] J. W. Pitman and M. Yor. Some divergent integrals of Brownian motion. Adv. in Appl. Probab., (suppl.):109-116, 1986.

[18] D. Revuz and M. Yor. Continuous martingales and Brownian motion, volume 293 of Grundlehren der Mathematischen Wissenschaften. Springer-Verlag, Berlin, third edition, 1999.

[19] C. Stone. Limit theorems for random walks, birth and death processes, and diffusion processes. Illinois J. Math., 7:638-660, 1963.

[20] S. Watanabe. Generalized arc-sine laws for one-dimensional diffusion processes and random walks. In Stochastic analysis (Ithaca, NY, 1993), volume 57 of Proc. Sympos. Pure Math., pages 157-172. Amer. Math. Soc., Providence, RI, 1995. 
[21] D. Williams. Path decomposition and continuity of local time for one-dimensional diffusions. I. Proc. London Math. Soc. (3), 28:738-768, 1974.

[22] X. X. Xue. A zero-one law for integral functionals of the Bessel process. In Séminaire de Probabilités, XXIV, 1988/89, volume 1426 of Lecture Notes in Math., pages 137153. Springer, Berlin, 1990.

[23] K. Yano. Excursion measure away from an exit boundary of one-dimensional diffusion processes. Publ. RIMS, 42-3:837-878, 2006. 